ISII I)IO ISSPI: III.

\title{
ENCUESTAS SOBRE TASAS DE PORTADORES SANOS DE N. MENINGITIDIS Y CARACTERIZACIÓN DE CEPAS CIRCULANTES
}

\author{
Julián Mauro Ramos Aceitero \\ Red de Vigilancia Epidemiológica, Dirección General de Salud Pública. Consejería de Sanidad y Consumo. Junta de Extremadura.
}

\section{RESUMEN}

Fundamento: Durante la temporada epidérnica 1996-97 el incremento de incidencia de Enfenmedad Meningocócica por N. Meningitidis serogrupo $C$ llevó a instaurar programas de vacunación y realizar distintos estudios, entre ellos dos para conocer la prevalencia de portadores sanos que presentamos.

Método: Portadores sanos en población general: encuesta transversal realizada entre marzo y mayo de 1998, entre residentes en Extremadura. Tomamos dos grupos de edad: $0-19$ años, vacunados $(n=1.140)$ y 20 y más afios $(n=1.193)$, no vacunados. Portadores sanos en un acuartelamiento: encuesta transversal en una base militar durante mayo de $1998(n=619)$, tomando dos grupos: $<20$ afos $(n=453)$ vacunados, y 20 y más años ( $\mathrm{n}=166)$ no vacunados.

Resultados: Portadores sanos en población general. El porcentaje de portadores para todos los serogrupos fue: grupo de 0-19 años 7'98, grupo 20 y más aftos 3'32, total 4'60, para serogrupo B: 4'43, 2'25 y 2'84; para C: 0'04, 0' 10 y $0^{\prime} 08$ respectivamente. Las diferencias entre ambos grupos de edad del serogrupo B y total son estadísticamente significativas, ast como la mayor prevalencia del $\mathrm{B}$ sobre $\mathrm{C}$. El subtipo de las tres muestras con aislamiento de serogrupo $C$ fue: una cepa NT:P1.6, una 2b:P1.2, y una no pudo sub-tiparse. Una cepa no serogrupable se subtipo como $2 \mathrm{~b}$ : P1. 5. Portadores sanos en un acuartelamiento. El porcentaje de portadores para todos los serogrupos fue: $<20$ años $8^{\prime} 43,20$ y más años $6^{\prime} 84$, total 7'27, para serogrupo B: 4'82, 3'75 y 4'04; y para C: 0'60, 0'00 y 0'16 respectivamente. Las mayores prevalencias obtenidas en militares sobre población general mostraron diferencias estadísticamente significativas. También la prevalencia de serogrupo B es significativamente muy superior a la C. La única cepa de $C$ aislada se subtipó $2 b$ : p1.2,5.

Conclusiones: La prevalencia de portadores sanos de $N$. mening $i$ tidis en población general es de 4,60\%, siendo mayor en el grupo de menor edad y el acuartelamiento. Muy escasa prevalencia del serogrupo $C$. Imposible concluir que cepa $N$. meningitidix serogrupo $C$ circulaha la temporada 1997198 .

\section{ABSTRACT \\ Survey of Rates of Healthy Carriers of N. Meningitidis and CharacterisatioN of Circulating Strains}

\begin{abstract}
Background: During the epidemic season of 1996-97, the increase in the incidence of Meningococcal Disease caused by $N$. Meningitidis serogroup $C$ led to the initiation of vaccination programmes and to the performance of a number of studies, among which, two were designed to ascertain the prevalence of healthy carriers and are presented here.

Methods: Healthy carriers in the population at large: cross survey performed between March and May 1998, among residents in Extremadura. We took two age groups: $0-19$ years of age, vaccinated $(n=1,140)$ and 20 years of age and over $(n=1,193)$, unvaccinated. Healthy carriers in a military barracks: cross survey on a military base in May 1998 $(n=619)$, taking two groups: $<20$ years of age $(n=453$ ) vaccinated, and 20 years of age and over $(n=166)$ unvaccinated.

Results: Healthy carrier in the population at lange. The percentage of carriers for all of the serogroups was: 0-19 years of age 7.98, 20 years of age and older 3.32 , total 4.60 , for serugroup $B: 4.43,2.25$ and 2.84 ; for $C: 0.04$, 0.10 and 0.08 respectively. The differences between the two age groups in serogroup $B$ and the total are statistically significant, as well as the greater prevalence of $B$ over $C$. The subtype of the three samples with isolation of serogroup $C$ was: one strain NT:P1.6, another strain $2 \mathrm{~b}: \mathrm{P} 1.2$, and one that coukd not be sub-typed. A strain that could not be classified into a serogroup was sub-typed as $2 \mathrm{~b}$ : P1. 5. Healthy carriers in a military barracks. The percentage of camiers for all of the serogmuns was: $<20$ years $8.43,20$ years of age and over 6.84 , total 7.27, for serogroup $B: 4.82,3.75$ and 4.04 ; and for $C: 0.60,0.00$ and 0.16 respectively. The higluer prevalence obtained in military subjects in comparison with the population at large revealed statistically significant differences. Also, the prevalence of serogroup B was significantly higher than C. The only isolated C strain was sub-typed as: p1.2.5.

Conclusions: The prevalence of healthy carriers of $N$. meningitidis in the population at large was $4.60 \%$, being higher in the lower age group and the barracks. Very low prevalence of serogroup C. Impossible to conclude that the strain $N$. meningitidis serogroup $C$ was circulating during the $1997 / 98$ season.
\end{abstract}

Correspondencia:

Julián Mauro Ramos Aceitero

Consejería de Sanidad y Consumo.

Dirección General de Salud Pública.

C/ Adriano, $n^{\circ} 4.06071$.

Mérida (Badajox).

Telf. 927.38 .12 .10

\section{INTRODUCCIÓN}

Como el resto del país, durante la temporada epidémica de1996-97 en Extremadura asistimos a un importante incremento de la incidencia de Enfermedad Meningocócica 
por el serogrupo $\mathrm{C}$ de $N$. meningitidis. Esto llevó a instaurar un programa especial de vacunación frente a $N$. meningitis serogrupos A-C, y a plantearnos, como otras CC.AA, la realización de algunos estudios para intentar acercarnos al conocimiento de la epidemiología del meningococo C. Estos estudios se concretaron en tres: un primer estudio sobre la efectividad de la seroconversión de la vacuna utilizada, cuya metodología fue presentada en la primera jornada sobre Enfermedad Meningocócica en febrero de 1998, y que ha tenido resultados similares a otros estudios aquí presentados; un segundo estudio, también presentado en aquella primera jornada sobre la Enfermedad Meningocócica, destinado a intentar conocer la tasa de portadores de $N$. meningitidis en población general; y un tercer estudio, similar al anterior, pero realizado en una base militar (Botoa, Badajoz) que nació justamente tras la presentación de aquel en dicha jornada, al ofrecernos el representante del Instituto de Medicina Preventiva del Ejército, la posibilidad de realizarlo. Los resultados obtenidos en estos dos estudios sobre prevalencia de portadores sanos son los que se presentan en este trabajo.

\section{MATERIAL Y MÉTODO}

\section{Estudio de prevalencia de portadores sanos en población general}

Como he dicho, la metodología del estudio ya fue presentada en su día, por lo que sólo a título de recuerdo diremos que se trató de una encuesta transversal, cuyo trabajo de campo se realizó entre los meses de marzo y mayo de 1998. La población diana la constituían los residentes en Extremadura a fecha 31 de diciembre de 1997, con tarjeta sanitaria. Se dividió en dos grupos de edad: de 0 a 19 años, y de 20 y más años, ya que la campaña de vacunación había sido realizada en niños y jóvenes de 18 meses a 19 años de edad, y queríamos ver posibles diferencias en la prevalencia de portadores y/o en las ce- pas circulantes en ambos grupos de población.

La muestra se obtuvo de los listados de tarjeta sanitaria facilitados por las Gerencias de Atención Primaria del Insalud, mediante un muestro por conglomerados bietápico no equiprobabilístico, eligiendo en una primera etapa 24 zonas básicas de salud de las 96 que entonces constituían la Comunidad Autónoma; y en una segunda etapa un número fijo de 50 personas para cada grupo de edad en cada zona. Es de destacar que para el cálculo del tamaño muestral el nivel de significación se marcó en el $90 \%$, en lugar del $95 \%$ habitual, por razones operativas, de forma que el tamaño muestral resultante fuera asumible.

Así, partiendo de una prevalencia esperada de portadores del $30 \%(\mathrm{P}=0$ '3), un máximo error admisible absoluto del $3 \%$, que representa el $10 \%$ de error relativo (e = 0 '03), el nivel de significación ya indicado del $90 \%(p=0$ ' 10), y un efecto de diseño de $1 ' 5$, el tamaño muestral fue de 631 y 946 en los grupos de edad de 0 a 19 y de 20 y más años respectivamente, que se aumentó definitivamente a 1.200. Por la experiencia con el estudio de seroconversión, se tomó un número muy superior de posibles participantes, para poder asumir altas tasas de no respuesta y/o participación. En definitiva, se eligieron 2.400 personas de cada grupo de edad a razón, como he dicho, de 50 personas por cada una de las 24 zonas de salud elegidas.

Las muestras biológicas de exudado rinofaríngeo se tomaron en los Centros de Salud con sembrado directo en medio de cultivo específico y trasladada posteriormente en contenedores con ambientc anaerobio y en menos de 3 horas a los tres laboratorios de la Comunidad Autónoma que participaron: el del Hospital Clínico Universitario «Infanta Cristina» de Badajoz, y los dos Laboratorios de Salud Pública de los Servicios Territoriales de la Consejería de Sanidad y Consumo, en los que se procesaron hasta el aislamiento de $N$. meningitidis. Posteriormente, las cepas aisladas se remitieron al Centro de referencia nacional de meningococos de Majadahonda 
dependiente del Instituto de Salud Carlos III, donde se confirmaba el aislamiento y, en su caso, se identificaba la cepa aislada.

\section{Estudio de prevalencia de portadores sanos en militares de un acuartelamiento}

El estudio, también transversal, se realizó en militares de la base de Botoa y se llevó a cabo durante el mes de mayo de 1998. Se estudiaron un total de 619 militares tanto profesionales como de reemplazo, pertenecientes a las unidades de «combate». En esta base militar también se había procedido a vacunar frente a $N$. meningitidis $\mathrm{A}$ y $\mathrm{C}$ a los menores de 20 años al comienzo de la temporada epidémica de 1997-98. Por ello la población estudiada se dividió en dos grupos: el de menores 20 años que había recibido la vacuna, y el de 20 y más años que no la había recibido. De esta manera intentamos conocer el posible efecto de la vacuna sobre el estado de portador, asumiendo que la edad puede comportarse como factor de confusión. No se realizó muestreo, sino que se estudió toda la población diana, por lo que no se muestran Intervalos de Confianza, es decir: no pretendemos inferir estos datos para la población general ni para otras unidades militares. La toma y análisis de las muestras biológicas se realizó de la misma forma que en el estudio poblacional.

\section{RESULTADOS}

\section{Estudio de prevalencia de portadores sanos en población general}

La tasa de participación fue cercana al 50\% en ambos grupos de edad, concretamente del $47 \%$ en el grupo de menor edad, y del $49 \%$ en el de mayor edad.

Las edades medias y porcentaje por géneros fueron las que se muestran a continuación:

Edad media (desviación estándar):

- $\quad$ Grupo de edad de 0 a19 años: 11,8 $(4,7)$.

- Grupo de edad de 20 y más años: $50,9(17,68)$

Porcentaje por género:

- Grupo de edad de 0 a19 años: mujeres: $53,1 \%$; hombres $46,9 \%$.

- Grupo de edad de 20 y más años: mujeres: $65,0 \%$; hombres $34,0 \%$.

La prevalencia de portadores encontrada fue la indicada en la tabla 1:

Tabla 1

Prevalencia de portadores sanos en población general. Porcentajes por edad y serogrupo (IC 90\%)

\begin{tabular}{|c|c|c|c|}
\hline & \multicolumn{3}{|c|}{ Grupos de Edad } \\
\hline Serogrupo & $\begin{array}{l}0-19 \text { años } \\
(n=1.140)\end{array}$ & $\begin{array}{l}20+a \tilde{n} o s \\
(n=1.193)\end{array}$ & $\begin{array}{l}\text { Prevalencia total } \\
\qquad(n=2.333)\end{array}$ \\
\hline B & $4,43(3,5-5,5)\left(^{*}\right)$ & $2,25(1,5-3,1)\left(^{*}\right)$ & $2,84(2,3-3,4)$ \\
\hline $\mathrm{C}$ & $0,04(0,0-0,3)$ & $0,10(0,0-0,4)$ & $0,08(0,0-0,2)$ \\
\hline A & 0,00 & 0,00 & 0,00 \\
\hline Otros & $0,44(0,1-0,9)$ & $0, \infty 0$ & $0,12(0,0-0,3)$ \\
\hline No identificados & $3,07(2,3-4,0)(*)$ & $0,97(0,5-1,6)(*)$ & $1,55(1,1-2,0)$ \\
\hline Total & $7,98(6,7-9,4)\left(^{*}\right)$ & $3,32(2,5-4,3)(*)$ & $4,60(3,9-5,3)$ \\
\hline
\end{tabular}


Las diferencias en las prevalencia entre ambos grupos de edad de los serogrupos B, no identificados y la prevalencia total $\left(^{*}\right)$, son estadísticamente significativas. También son significativamente mayores las prevalencias del serogrupo B sobre el C, en ambos grupos de edad y en el total. Las prevalencias totales pueden considerarse dentro de lo resultados indicados en otros estudios, sobre todo por la gran variabilidad de esos resultados y de los propios estudios; mientras que la prevalencia de portadores del serogrupo C, en el grupo de menor edad está por debajo de lo encontrado en el estudio realizado en Galicia en la temporada 1996/97.

En cuanto al intento de caracterizar las cepas circulantes, resulta difícil por el escaso númcro de cepas aisladas de $N$. meningitidis serogrupo $\mathrm{C}$, tan sólo 3 , lo que hace que los datos sean muy pobres. Estas 3 muestras procedían de Zonas de Salud del norte de la provincia de Cáceres. En esta área geográfica se registraron los primeros casos de enfermedad Meningocócica por serogrupo $\mathrm{C}$ en nuestra Comunidad Autónoma, hacia 1990, llegando a aparecer un brote por este serogrupo C en 1992 que llevó a la utilización de la vacuna por primera vez en Extremadura.

El subtipo de estas tres muestras fue:

- una cepa del subtipo NT:P1.6.

- una del 2b:P1.2.

- una tercera cepa no pudo sub-tiparse.

Por otra parte, una de las cepas no serogrupables, por aglutinar con varios tipos de sueros de $N$. meningitidis, se subtipo como 2b: P1. 5.

\section{Estudio de prevalencia de portadores sanos en militares de un acuartelamiento}

Los resultados obtenidos se muestran en la tabla 2.

Tabla 2

Prevalencia de portadores sanos en militares de la base de Botoa. Porcentajes por edad y serogrupo

\begin{tabular}{|l|c|c|c|}
\hline & \multicolumn{3}{|c|}{ Grupos de Edad } \\
\hline Serogrupo & $\begin{array}{c}|c| \\
(n=453)\end{array}$ & $\begin{array}{c}20+a \tilde{o} \text { s } \\
(n=166)\end{array}$ & $\begin{array}{c}\text { Total } \\
(n=619)\end{array}$ \\
\hline B & 4,82 & 3,75 & 4,04 \\
C & 0,60 & 0,00 & 0,16 \\
A & 0,00 & 0,00 & 0,00 \\
Otros & 0,60 & 0,22 & 0,32 \\
No identificados & 2,40 & 2,39 & 2,74 \\
Total & 7,43 & $6,84(* *)$ & $7,27(* *)$ \\
\hline
\end{tabular}

Al igual que ocurre con la población general, la prevalencia de portadores en el grupo de menor edad es mayor que en el grupo de más edad. Sin embargo, en este caso estas diferentes proporciones de portadores sanos entre los dos grupos de edad no mostraron significación estadística. Sin embargo las mayores prevalencias para todos los sero- grupos obtenidas en militares con respecto a población general en el grupo de mayor edad y en el total de población (**), si mostraron diferencias estadísticamente significativas. $Y$ también, en el caso de los militares, la prevalencia de portadores del serogrupo B es significativamente muy superior a la del serogrupo $\mathrm{C}$. 
La única cepa de $N$. meningitidis serogrupo $\mathrm{C}$ aislada se subtipó como $2 \mathrm{~b}: \mathrm{p} 1.2,5$.

\section{CONCLUSIONES}

Para terminar, las únicas conclusiones que creo podemos obtener de estos resultados son:

- La prevalencia de portadores sanos de $N$. meningitidis en población general del 4,60\%.
- Se observa una mayor prevalencia en grupo de menor edad.

- Se observa una mayor prevalencia en el acuartelamiento.

- Hay una muy escasa prevalencia de portadores del serogrupo $\mathrm{C}$.

- Con los pocos datos sobre cepas no nos atrevemos a concluir nada sobre que cepa $N$. meningitidis era la que teníamos circulando durante la temporada $1997 / 98$. 